\title{
Learning and Transfer: Instructional Conditions and Conceptual Change
}

\author{
Michelle Perry \\ University of Michigan
}

\begin{abstract}
It is widely assumed that instruction plays a role in learning and in transfer. The present studies examine how type of instruction (containing principle-based vs. procedure-based information) influences learning and transfer in a mathematical concept. In the first study, both types of instruction led a comparable number of children to learn, but principle-based instruction led significantly more children to transfer their new knowledge. In the second study, the types of instruction were combined (i.e., children received both principle and procedure information). The results were virtually identical to the results obtained from the procedure-only instructions. This indicates that principle-based instruction may be crucial for transfer to occur and, when children also are exposed to procedures, few will transfer. It is hypothesized that children may ignore the conceptually rich information inherent in the principle when procedures are also provided.
\end{abstract}

How children acquire new knowledge is a problem faced by cognitive and developmental psychologists, educators, and even parents. This is an important problem because it has implications, theoretically, for how the mind works and, practically, for helping children to learn. This problem, however, is multifaceted and could be investigated from many different angles. The part of the problem that is examined here is how various instructional conditions influence knowledge acquisition.

Ideally, instruction would affect not only learning, but also transfer. As Gick and Holyoak (1987) have pointed out, learning can take place anywhere along a continuum from "ordinary learning" (Osgood, 1949), or performance on new instances of the same task, to "near transfer" (e.g., Brown \& French, 1979), or

Portions of this article were presented at the AERA annual meeting in New Orleans and at the biennial meeting of ISSBD in Jyvaskyla, Finland. This work was funded in part by a Rackham Faculty Research Grant (\#386155) and in part by an NICHD Grant (\#R0I HD18617). I would like to thank the principals, teachers, and students who made this project possible. I would also like to thank Jeff Bisanz, Breckie Church, Susan Gelman, Susan Goldin-Meadow, Terri Graham, Karen Hinnegan, John Ifcher, George Kamberelis, Steven LaCommare, Katherine Nelson, Jim Stigler, and Tom Trabasso and an anonymous reviewer for their input at various stages of this research.

Correspondence and requests for reprints should be sent to Michelle Perry, University of Michigan, Developmental Psychology, 34433 Mason Hall, Ann Arbor, MI 48109-1027. 
performance on similar tasks that require the same rules for solution, to "far transfer," or performance on dissimilar tasks that require similar rules.

One goal of the current research is to specify the degree of learning that has taken place. Another goal is to examine the effects of different types of instruction on learning and transfer, where learning is defined as success on similar problems and transfer as success on dissimilar, but conceptually related problems (Chi \& Bassok, 1989). It generally has been argued that conceptually based or abstract instruction provides more benefits for the learner than procedurally based or concrete instruction (Hiebert \& Lefevre, 1986). However, findings are not conclusive. Thus, the present research will begin to explain why it is that not all instructional attempts produce learning and even fewer allow transfer to new, but conceptually related tasks in the domain of mathematics.

Different factors are presumed to promote or facilitate transfer. The work presented here is concerned with the way in which instruction directly influences learning and transfer. The question of what type of instructional information is most likely to lead to learning and transfer is a classic one. Much of the debate has focused on whether procedural instruction is better than principled instruction or whether principled instruction is better (see, e.g., Hiebert, 1986). In essence, this distinction centers on whether it is better to inform learners exactly how to solve some problem (the procedural approach) or why a problem should be solved in a particular way (the principled approach). Among the first psychologists to debate this issue were Thorndike and Judd. Thorndike and Woodworth (1901) argued that surface features must be equivalent across problems for transfer to occur. Judd (1908), on the other hand, reported that transfer is dependent upon having a guiding principle, and that principles can be provided in instruction. Although Thorndike (1926) later withdrew his position, Judd's position that transfer could be promoted if subjects were instructed in a guiding principle was not a widely held position.

This issue has continued to receive attention throughout the century. Over 30 years ago, Corman (1957) examined whether success in transfer was enhanced by more, less, or no information given as guidance. In general, he found that information about a procedure for solving the problems led to success on posttest problems, and that success on posttest problems led to success on transfer problems.

However, this does not necessarily mean that instruction in the procedure is always the best form of instruction. The fact that success on the posttest and the transfer problems was so closely linked suggests that the transfer problems were not very different from the posttest problems. As a further point, Corman (1957) examined a concrete problem (i.e., in a particular configuration of matches, move three matches to change the configuration from five squares to four squares) in which the subjects had no experience. This indicates that although information about a problem-solving procedure was helpful, no conclusions can be drawn about what type of instructional information would be helpful for more abstract problems or for problems in which the domain was more familiar. 
More recently, Brown and Kane (1988) have looked at the transfer abilities of preschool children. They examined children's abilities to apply a rule (e.g., about mimicry in nature - that a caterpillar, which looks like a snake, is protected from being eaten by birds) to new examples. They found that young children were capable of learning a general principle and then applying that principle to other examples. Further, the best method for enabling children to perform well was to have children verbalize the principle (either spontaneously or after the rule was explicitly mentioned by the instructor). This result contradicts Corman's (1957) result that a problem-solving procedure is the best form of instruction. Unlike Corman's work, in Brown and Kane's work, principle instruction was helpful for very young children and the problems were more typical of school-type and everyday-type problems than of arbitrary test items. Brown and Kane's work would suggest that exposing children to a principle is a powerful form of instruction.

In another recent study, Perry, Church, and Goldin-Meadow (1988) provided 10-year-old children with a principle-based instruction for solving mathematical equivalence problems. An example of this type of problem is: $4+6+9=-+$ 9. At the onset of the study, none of the children solved any of the test problems correctly. The principle instruction was effective for $46 \%$ of all children. Moreover, the principle-based instruction led to children's success not only on the type of problems on which they were instructed, but also on transfer problems. In fact, $82 \%$ of those children who demonstrated any learning were successful on every type of problem after instruction.

A shortcoming of these studies with respect to the problem at hand is that none directly compares procedure- to principle-based instruction. Some studies provide evidence supporting the argument that a principled approach is important for learning to take place, whereas others provide evidence supporting the argument that a procedural approach is important for learning to take place. Empirical evidence is needed that is based on a direct comparison of these instructional approaches. The studies reported herein have been designed to compare these two types of instruction, and therefore to establish a systematic basis for future instructional interventions.

In Study 1, Perry et al.'s (1988) work was extended, by repeating the principle-based instruction with new subjects and adding a procedure-based instruction as a comparison. In Study 2 , the combined effects of principle-based and procedure-based instruction were investigated.

\section{STUDY 1}

\section{Method}

Subjects. Forty-one fourth- and fifth-grade children ( 24 boys and 17 girls) participated in all phases of the study. Children were randomly assigned to one of 
two instructional conditions: principle alone $(n=10)^{\prime}$ or procedure alone $(n=$ 31). The children were sampled from nine different schools. Each of the schools was a private parochial school. Six of the schools were located in residential neighborhoods within a large midwestern city, and three schools were located in the suburbs of another large midwestern city.

Materials. The difficulty of the task was an important consideration in devising the problems used to test learning and transfer. Previous work (Perry, 1985; Perry et al., 1988) has identified a set of problems that most fourth- and fifth-grade children (approximately $90 \%$ ) failed. It was important that most children spontaneously solved the problems incorrectly so that children could be equated for initial knowledge of the problems.

Additionally, it was important that the task format was familiar, and that all children had necessary prerequisite knowledge so that they had comparable chances of succeeding on learning how to solve the problems. The mathematics problems chosen for study represented the principle of mathematical equivalence (i.e., the principle that the sum of the numbers on one side of an equation is equal to the sum of the numbers on the other side of the equation). An example of the type of problem that was used is: $4+6+9=\ldots+9$. In this problem, "10" is the correct solution because that is the quantity that will make both sides equal. Several more simple addition problems (e.g., $6+5=\ldots$ ) were included on the pretest sheet, so that any child who could not add could be eliminated from the study (thus, satisfying the need that all children be equated for prerequisite knowledge). No child exhibited difficulties with simple addition.

A pretest was constructed of six problems (of the form " $a+b+c=-+c$ " or " $a+b+c=a+\ldots$ ). These pretest problems can be found in Table 1 . These problems have been found to provide an indication of children's depth of knowledge about the equal sign because children who could solve more simple addition problems could not solve the more complex problems correctly (Perry, 1985). For example, children aged 9 to 12 are capable of solving problems with multiple addends that all appear on one side of the equation (e.g., $4+6+9=$ -) and, in problems like this, appear to understand mathematical equivalence. However, children may be getting the correct answer for the wrong reasons. It has been suggested (e.g., Behr, Erlwanger, \& Nichols, 1980; Kieran, 1980) that children may interpret the equal sign as an instruction to add all of the numbers on the left side of the equation and not as an instruction to make both sides of the equation equal.

Also note (from Table 1) that each side of the equation summed to a total of $\leq$ 19 to reduce the likelihood that any of the children would encounter difficulty in

\footnotetext{
1 Only 10 children were assigned to this condition because this condition was included to replicate some of the findings reported in Perry et al. (1988). Where applicable, relevant reanalyses of the 37 children from Perry et al. (1988) are presented in the text.
} 
Table 1. Addition Problems Used on the Pretest

$$
\begin{aligned}
& 4+6+9=-+9 \\
& 7+6+5=-+5 \\
& 3+7+9=-+9 \\
& 2+5+9=2+- \\
& 7+4+2=7+- \\
& 3+6+8=3+-
\end{aligned}
$$

adding, which is more common when children work with large numbers (Brown \& Burton, 1978). This was done so that it would be more likely that errors could be attributed to not understanding that both sides of the equation should be equal, rather than problems such as "buggy algorithms." To further assure that understanding of the equal sign was at issue, and not a child's problems with the addition operation, a problem was coded as correctly solved if the number placed in the blank came within \pm 2 of the correct solution. For example, in the problem $4+6+9=\ldots+9$, if a child placed an 11 in the blank, the error would be noted as an addition error, but the child was credited with using a correct strategic approach to the problem. This was done because, for the purposes of this study, the strategy used to arrive at the solution was important, but the precision of the solution was not.

Procedures. Each child participated in a series of four consecutive sessions conducted on the same day. The sessions were held in an empty classroom or other available space in the school (e.g., library) and each child was tested individually. The sessions included pretest, instruction, posttest, and transfer sessions, described as follows.

During the pretest, each child was given a paper-and-pencil addition test (see Table 1). Children were asked to solve the problems, which were presented on a sheet of paper, on their own. After the child completed the test, an experimenter wrote the first problem along with the child's solution to the problem, on a blackboard and asked the child to explain to her how he or she arrived at that solution. This procedure was repeated for the remaining five problems on the test. The two children who solved any of these six pretest problems correctly were excluded from further participation and analysis. The explanations of the remaining children were coded into strategies and these data are described in detail in Perry et al. (1988).

During instruction, the child was exposed to either the principle underlying the addition problems or a step-by-step procedure for solving the addition problems. An experimenter who was not at the pretest, and therefore who was blind to the types of explanations the child produced on the pretest, randomly assigned the child to one of the instructional conditions.

In the principle instruction condition, the experimenter wrote on the black- 
board an addition problem of the sort that was on the paper-and-pencil test. The experimenter instructed the child in the principle underlying the problem at hand; specifically, she told the child: "The goal of a problem like this is to find a number that fits in the blank that makes both sides equal; that is, to make this side [pointing to right side of equation] equal to this side [pointing to left side of equation]." Children were allowed to ask questions and, if anything remained unclear, the experimenter repeated the instructions. However, no instruction was given in any procedures for achieving this goal ${ }^{2}$ and no solutions were provided.

After showing the first problem, the experimenter wrote a second problem on the blackboard and asked the child to attempt to solve this problem. The experimenter gave no feedback to the child (other than general encouragement), but would repeat the instructional phrases if the child requested assistance. Up to this point, the child had heard the experimenter give instruction for one problem and was allowed to solve one problem. The experimenter repeated this sequence with two addition problems of the same type. In other words, the experimenter gave the same instructions, first without allowing the child to solve the problem, and then with giving the child a new problem to try to solve. Thus, the child heard instructions at least twice and solved two problems.

One could argue that these instructions reveal more information about the goal than about the principle of equivalence, simply because the word 'goal' was used in the instructions. However, the word 'goal' was chosen because, in pilot work, children were able to focus on the principle of equivalence when the experimenter used 'goal,' but not when she used 'principle' (perhaps because they were more familiar with the term 'goal').

In the step-by-step procedure instruction condition, the experimenter wrote the same addition problem that was used in the principle instruction condition on the blackboard and explained a step-by-step procedure for solving the problem to the child. Specifically, the experimenter told the child: "One way to solve a problem like this is to add up all the numbers on the left side of the problem (e.g., in the problem $4+6+9=\ldots+9$, the experimenter would use her hand to indicate the $4+6+9$ ) and then subtract the number on the right side of the problem (and then indicate the 9 on the right side of the equation)." This instructional strategy was used because the experimenter could easily avoid articulating the principle of equivalence in her explanations, and because the few children who demonstrated understanding of these problems tended to rely on this particular strategy (i.e., in pilot work, children appeared to arrive at this strategy spontaneously). The experimenter did not mention the principle underlying the

\footnotetext{
2Admittedly, the label principle is imperfect. Some might even argue that some procedures (i.e.. make both sides equal) were implicit in this condition. Still, how to carry out this procedure (if, indeed, one chooses to call this a "procedure") is not specified. It is important to note that although commonalities exist between the instructional conditions, differences exist as well, and it is these differences that are highlighted in the terms principle and step-by-step procedure.
} 
use of this procedure, nor did she provide a solution to the problem. The experimenter then wrote a second problem on the blackboard and asked the child to attempt this problem on his or her own. The experimenter gave no feedback to the child (other than general encouragement), but would repeat the instructional phrases if the child requested assistance. Up to this point, the child had heard the experimenter give instruction for one problem and was allowed to solve one problem. The experimenter repeated this sequence with the same two addition problems used in the latter part of the principle instruction condition. Thus, the child heard instruction at least twice and solved two problems.

During the posttest, each child was given a paper-and-pencil test that contained six addition problems comparable to those on the pretest. As in the pretest, after the child completed the paper-and-pencil test, the experimenter had the child explain each of his or her solutions at the blackboard.

During the transfer test, each child was asked to solve two problems, instantiating a new operation, multiplication (e.g., $2 \times 4 \times 3=2 \times-$ ), and eight problems designed to determine which solutions the child would consider acceptable answers to a particular problem (e.g., "If you were the teacher and a student put a 13 in the blank for the problem $4+6+3=\ldots+3$, would you tell the student that his or her answer was correct or incorrect? Why? How about if the student put a 10 in the blank?" etc.). All of the transfer problems can be found in the Appendix. These types of problems did not appear on the pretest so that transfer, as opposed to learning, could be measured. As Gick and Holyoak (1987) point out, "in transfer paradigms the second task is typically selected to be novel to the subject" ( p. 10). In essence, these problems were used because the goal was to have problems that tested children's understanding of equivalence beyond the context in which the children were tested and instructed.

For all of the transfer problems, the principle of having both sides of the equation equal the same total could guide the children to correct solutions. However, the manner in which this principle was realized-that is, the procedure used to solve these problems-could differ across problems and/or children.

\section{Results and Discussion}

The results are discussed in two sections. The first deals with effects of type of instruction on posttest performance (i.e., on learning) and the second deals with effects of instruction on ability to transfer knowledge.

Effects of Instruction on Posttest Performance. The question addressed here is: Which, if any, of the types of instruction were successful in promoting learning? Further, even if both led to some success, was one type of instruction more successful than the other?

Overall, $45 \%$ of the children (i.e., 20 out of 44) were successful on the posttest. The criterion for success was defined as solving at least five of the six posttest problems correctly (i.e., children were allowed to commit one error and 
still be given credit for understanding how to solve these problems). The reason for using this criterion (i.e., successful or unsuccessful, as opposed to number of problems solved correctly) was that there was a bimodal distribution of number of problems solved correctly: $90 \%$ of the children either solved none of the problems correctly or solved five or all six of the problems correctly. The small proportion of children solving only some, as compared to none or all, of the posttest problems correctly lends validity to this criterion of success. Table 2 shows the proportion of children, separately for the two instructional conditions, who were successful on the posttest. As can be seen in Table 2, the percentage of children who solved the posttest problems correctly ranged from $40 \%$ (in the principle instruction condition) to $47 \%$ (in the procedure instruction condition).

The children from Perry et al.'s (1988) report performed similarly: $43 \%$ of those children were successful on the posttest. Recall that all of the children in that study were exposed to the principle. These data are also presented in Table 2.

A Chi-square test found no significant differences in the effects of type of instruction on learning, $\chi^{2}(1, N=44)=.16, p>.10$. Thus, the two instructional conditions were indistinguishable in terms of promoting children's learning, as measured by correct performance on the posttest.

Effects of Instruction on the Transfer Problems. The question posed here is: Which, if any, of the types of instruction were successful in enabling children to transfer their knowledge to new types of problems?

Overall, only $16 \%$ of the children (i.e., 7 out of 44 ) were successful on the transfer problems (e.g., multiplication problems such as $2 \times 3 \times 4=-\times 4$ ). The criterion for success was defined as solving all, or all but one, of the transfer problems correctly. Specifically, children were allowed to commit one error only if that type of problem appeared at least twice (i.e., children were allowed to commit one error only if they could demonstrate that they understood the princi-

Table 2. Percentage of Children Succeeding After Instruction (Study 1)

\begin{tabular}{lccc}
\hline & \multicolumn{3}{c}{ Instructional Condition } \\
\cline { 2 - 4 } \multicolumn{1}{c}{ Degree of Success } & Procedure & Principle & $\begin{array}{c}\text { Principle } \\
\text { (from Perry et al.) }\end{array}$ \\
\hline No success on posttest problems & $53 \%$ & $60 \%$ & $57 \%$ \\
Success on posttest problems & $47 \%$ & $40 \%$ & $43 \%$ \\
Success on posttest and transfer problems & $9 \%$ & $40 \%$ & $38 \%$ \\
$\boldsymbol{n}$ of children & 31 & 10 & 37 \\
\hline
\end{tabular}

Note. The percentages do not sum to 100 because children who demonstrated success on both the posttest and transfer problems are also represented in the group of children who demonstrated success on the posttest problems. Thus, the percentage of children who show no success plus the children who show success on the posttest problems sums to 100 . To determine the percentage of children who demonstrated success only on the posttest problems and not the transfer problems, subtract the number on the third line from the number on the second line. 
ple elsewhere on the transfer task). ${ }^{3}$ All but one child who correctly solved the transfer problems also correctly solved the posttest problems. As a point of interest, this one child solved four of the six posttest problems correctly, but was not considered to have demonstrated success on these problems because he did not meet the criterion of solving at least five correctly. Thus, with the exception of one child, it appears that children who correctly solved the transfer problems demonstrated true understanding of the concept of equivalence, in every way in which they were asked, whereas other children did not.

A Chi-square test was computed to identify whether there were significant differences between the types of instruction in producing success in solving the transfer and posttest tasks, combined. Although an analysis looking at success on the transfer problems separately could be done, this would not be a true test of children's ability to transfer what they learned. To be considered as having successfully transferred their knowledge, children had to demonstrate both that they solved the posttest problems correctly (i.e., they learned something) and solved the transfer problems correctly. Thus, this is a more conservative criterion of transfer than looking at the transfer problems independently of the posttest problems. Indeed, on this analysis, significant differences exist in the effects of type of instruction on transfer, $\chi^{2}(1, N=44)=5.61, p<.02$.

The proportion of children in each instructional condition who correctly solved the transfer problems, as well as the posttest problems, is conveyed in Table 2: The percentage of children who solved all of the transfer and posttest problems correctly was $9 \%$ for children in the procedure condition versus $40 \%$ for children in the principle condition. Again, the children in the principle condition performed similarly to the children reported by Perry et al. (1988): $38 \%$ of those children correctly solved the transfer problems. Thus, the children in the principle condition demonstrated an understanding of equivalence across all problems that they were given significantly more often than the children in the procedure condition.

It was possible, given the way that success was determined, that children could solve some, but not all, of the transfer problems correctly and still be classified as not successful. Thus, a few of the children were classified as not successful when they had solved some, but not all, of the transfer problems correctly. Whereas none of the children only solved the multiplication problems

\footnotetext{
${ }^{3}$ Only one type of problem was not given twice. This was the fifth alternative-solutions problem (see the Appendix): "If you were the teacher . . . and if Sam [your student] put $4+6$ in the blank for the problem $4+6+3=\ldots+3$, would you tell Sam that his answer was correct or incorrect?" Because this type of problem, with an addition problem as the solution, was only presented once, some leniency was observed in deciding whether children had committed an error. Children were given credit if they gave either a fully correct response (i.e., that Sam was correct) or a partially correct response (i.e., that although this was not the best answer, they, as the teacher, would not mark Sam's answer as incorrect). Children who said that $4+6$ was not correct were charged with an error on this problem.
} 
correctly, 8 children, all from the procedure condition, solved the alternativesolutions problems correctly (e.g., judged 10 and $4+6$, but not 13 , to be acceptable solutions to $4+6+3=\ldots+3$ ). If all 8 of these children had solved the posttest problems correctly, it may have been fair to call success on the alternative-solutions problems "near transfer" and success on the multiplication problems "far transfer." However, some of these children $(n=3)$ did not solve the posttest problems correctly and, because of this, could not be considered as being successful. Thus, the more stringent criterion of solving all of the transfer problems correctly was left in place for deciding transfer success because it appears that children who solved any of the transfer problems incorrectly really did not understand equivalence.

It is interesting to note how the children attempted to solve the transfer problems when their solutions were incorrect. For all of the children in the principle condition who did not solve the transfer problems correctly, their solutions were comparable to the incorrect solutions they produced on the pretest. For example, children who used the Add-All strategy (i.e., adding all of the numbers that appeared in the problem) on the pretest, used a Multiply-All strategy (i.e., multiplying all of the numbers that appeared in the problem) on the transfer multiplication problems. They also judged solutions as correct on the alternativesolutions component of the transfer test that could be derived from the strategies they used on the pretest. Perry et al. (1988) provided further details on how strategies were coded.

On the other hand, $42 \%$ of the children in the procedure condition who solved the transfer problems incorrectly $(n=31)$ did not revert to the incorrect strategies they used on the pretest. For the most part, these were the children who solved the posttest problems correctly, but did not solve the transfer problems correctly. These children correctly used the procedure that they were taught on the addition problems, adding the numbers on the left side of the equation and then subtracting the number on the right side of the equation. However, most of these children used a variation on the addition procedure for the multiplication problems. For these problems (e.g., $2 \times 3 \times 4=-\times 4$ ), they multiplied all of the numbers on the left side of the equation and then subtracted the number on the right side of the equation. Children's performance on the transfer problems pointed to the fact that very few of the children in the procedure condition actually understood mathematical equivalence; instead, these children were adept at applying a procedure, but only in analogous situations. Thus it appears that the children's responses indicated that the children forced an analogy where none existed.

Although the chances for success on the posttest problems were not significantly different for the children in the two conditions, the children in the principle condition were significantly more likely to demonstrate transfer. Also, children in the principle condition who solved any problem correctly after instruction were likely to solve all problems correctly after instruction. In contrast, if children in the procedure condition were to solve any problem correctly after 
instruction, they were likely to solve only the posttest problems correctly (and their understanding of the procedure is called into question, given the strange alterations in the procedure that were invented to accommodate multiplication problems). Thus, the results of this study imply that principle information is crucial for the rapid acquisition of a concept. Children receiving this sort of instruction demonstrate proficiency on all of the problems presented that test understanding of the concept of equivalence; that is, they both learn and transfer after receiving principle instruction.

Instruction in the procedure did not produce such impressive results. Although roughly half of the children could use the procedure to solve comparable addition problems, very few of these children could transfer this procedure to new types of problems that tested the same concept. Thus, procedure instruction led to relatively shallow learning (i.e., learning without transfer).

Two alternative hypotheses were generated from this finding: First, note that a larger percentage of children (albeit not significantly larger) receiving the procedure instructions solved the posttest problems correctly than children receiving the principle instruction. This leads to the hypothesis that if the procedure information is somehow easier to grasp, then the children who would not benefit from the principle information might be able to benefit from the procedure information. Thus, if the two instructions were combined, the children who may be unable to learn the principle could at least learn a procedure to solve some problems. Second, it could be that procedural information may distract children from understanding equivalence. In other words, children hearing the procedure might decide to use the procedure, but never analyze why the procedure works, nor under what conditions it can be used. Given this possibility, any child receiving procedure instruction would be at a disadvantage in solving the transfer problems, even if the principle information were available. These alternative hypotheses are examined in Study 2.

\section{STUDY 2}

The purpose of this study was to investigate whether the procedure instruction, when combined with principle instruction, could lead a greater proportion of children to solve posttest and/or transfer problems correctly than the procedure instruction alone or the principle instruction alone. Thus, the purpose of Study 2 is to investigate whether, when given both types of information, more children take advantage of the type of instruction that might be best suited for them.

\section{Method}

Subjects. Sixty-six fourth- and fifth-grade children (30 boys and 36 girls) participated in all phases of the study. Children were randomly assigned to one of two instructional conditions: principle-plus-procedure (i.e., principle informa- 
tion, followed by procedure information), or procedure-plus-principle. The children came from the same nine schools described in Study 1.

Procedures. As in Study 1, each child participated individually in a series of four consecutive sessions (i.e., pretest, instruction, posttest, and transfer) conducted on the same day. The five children who solved any of the pretest problems correctly were excluded from further participation and analysis. All four sessions were held in an empty classroom or other available space in the school (e.g., library). All children received both types of instruction that were described in Study 1. Some of the children received principle information before the procedure information, and some of the children received the procedure before the principle. This was done to allow analyses for recency and primacy effects that may interfere with interpretation of the results. Specifically, if only one of the orders were used and the results from that condition were in accord with the second hypothesis (and all children performed similarly to the children receiving the procedure instruction in Study 1), it would be impossible to know whether this was due to the general overwhelming effects of the procedure or to a recency or primacy effect (i.e., hearing the procedure last and focusing on only the procedure, or hearing the procedure first and tuning out the information about the principle that followed).

In the principle-plus-procedure instruction condition, the same four problems that were used in Study 1 were used. In this condition, the experimenter wrote the first problem that was used in Study 1 on the blackboard and instructed the child first in the principle and then in the procedure (using the exact training protocols from Study 1). The experimenter gave no feedback to the child (other than general encouragement), but would repeat the instructional phrases if the child requested assistance. The child then tried to solve a new problem (i.e., the second problem used in the instructional sequence, as described in Study 1). The experimenter repeated this sequence of instruction and then allowed the child to solve a different problem, as in Study 1.

In the procedure-plus-principle instruction condition, the same four problems that were used in Study 1 were used. In this condition, the experimenter wrote the first problem that was used in Study 1 on the blackboard and instructed the child first in the procedure and then in the principle (using the exact training protocols from Study 1). The experimenter gave no feedback to the child (other than general encouragement), but would repeat the instructional phrases if the child requested assistance. The child then tried to solve a new problem (i.e., the second problem used in the instructional sequence, as described in Study 1). The experimenter repeated this sequence of instruction and then allowed the child to solve a different problem, as in Study 1.

\section{Results and Discussion}

The results are divided into two sections. The first deals with effects of type of instruction on posttest performance and the second deals with effects on transferring knowledge. 
Effects of Instruction on Posttest Performance. The questions addressed here are: Was either type of instruction successful in promoting learning? Further, if success was found, was one type of instruction more successful than the other?

Overall, $59 \%$ of the children (i.e., 39 out of 66) were successful on the posttest. The criterion for success was the same as in Study 1 (i.e., children were allowed to commit up to one error and still be given credit for understanding how to solve these problems). Only $6 \%$ of the children solved between one and four problems correctly. As in Study 1, the small proportion of children correctly solving only some, as compared to none or all, of the posttest problems lends validity to this criterion of success. Table 3 shows the proportion of children, separately for the two instructional conditions, who were successful on the posttest. As can be seen in Table 3, the percentage of children who solved the posttest problems correctly was $56 \%$ for children in the principle-plus-procedure condition and $59 \%$ for children in the procedure-plus-principle condition.

A Chi-square test was computed to confirm that no significant differences existed between the instructional conditions in producing learning, $\chi^{2}(1, N=$ $66)=.19, p=.66$. Thus, the two instructional conditions were indistinguishable in terms of promoting children's correct performance on a set of similar addition problems on the posttest.

Effects of Instruction on the Transfer Test. The questions posed here are: Was either type of instruction successful in enabling children to transfer their knowledge to new types of problems? Further, if success were found, was one type of instruction more successful than the other?

Overall, only $11 \%$ of the children (i.e., 7 out of 66 ) were successful on the transfer problems. The criterion for success was the same as in Study 1 (i.e., children were allowed to commit one error only if they could demonstrate that they understood the principle elsewhere during the transfer task). Six of these 7 children also solved the posttest problems correctly, indicating that 6 children

Table 3. Percentage of Children Succeeding After Instruction (Study 2)

\begin{tabular}{lcc}
\hline & \multicolumn{2}{c}{ Instructional Condition } \\
\cline { 2 - 3 } \multicolumn{1}{c}{ Degree of Success } & Procedure-plus-Principle & Principle-plus-Procedure \\
\hline No success on posttest problems & $41 \%$ & $43 \%$ \\
Success on posttest problems & $59 \%$ & $56 \%$ \\
Success on posttest and transfer problems & $14 \%$ & $5 \%$ \\
$\boldsymbol{n}$ of children & 29 & 37 \\
\hline
\end{tabular}

Nore. The percentages do not sum to 100 because children who demonstrated success on both the posttest and transfer problems are also represented in the group of children who demonstrated success on the posttest problems. Thus, the percentage of children who show no success plus the children who show success on the posttest problems sums to 100 . To determine the percentage of children who demonstrated success only on the posttest problems and not the transfer problems, subtract the number on the third line from the number on the second line. 
(i.e., $9 \%$ of all the children in Study 2) demonstrated understanding of all of the problems that were presented to them after receiving instruction. Thus, it appears that (except for one child) children who solved the transfer problems correctly demonstrated true understanding of the concept of equivalence, in every way in which they were asked, whereas the remainder of the children in Study 2 did not.

The percentage of children in each instructional condition who correctly solved the transfer problems is shown in Table 3. A Chi-square test found no significant differences between the instructional conditions in producing success in solving the transfer problems, $\chi^{2}(1, N=66)=1.38, p=.239$. Thus, no evidence for recency or primacy effects was found. The percentage of children who solved the transfer problems correctly ranged from 5\% (in the principleplus-procedure condition) to $14 \%$ (in the procedure-plus-principle condition). Thus, as for the children in the procedure condition in Study 1, most children failed to demonstrate enough understanding of the principle of mathematical equivalence that would allow successful performance on the transfer problems.

As in Study I, although it seemed that some of the children in each of the instructional conditions had some chance of succeeding on the posttest, which was composed of problems that were similar to the pretest and instructional problems, none of the children who received procedural instruction were likely to succeed on the transfer problems. If children received any procedural information and were to solve any problem correctly after instruction, they were likely to solve only the posttest problems correctly.

Children who received the procedure-only instruction in Study 1 performed similarly to children in Study 2 who received both procedural and principle information. This is shown in Figure 1, which displays the percentage of children in all four conditions, and children from Perry et al. (1988), who succeeded only on the posttest or on both the posttest and the transfer problems. There are no statistically significant differences across the three conditions that included procedural information (i.e., procedure only, procedure-plus-principle, and principle-plus-procedure) for performance on the posttest, $\chi^{2}(2, N=97)=1.50, p=$ .47 , or for performance on the transfer task, $\chi^{2}(2, N=97)=1.40, p=.50$. Nor are there differences between the two conditions that included only principle information [i.e., principle-only from Study 1 and from Perry et al., $\chi^{2}(1, N=$ $47)=.03, p=.85]$. However, children in the principle-only condition performed significantly better on the transfer task than children in the three conditions containing procedure information, $\chi^{2}(3, N=110)=14.80, p=.002$, as did the children reported by Perry et al., who received principle information, $\chi^{2}$ $(3, N=137)=16.90, p<.001$.

Recall that the motivation for Study 2 was to investigate why children who received only the procedure instruction (in Study 1) demonstrated shallow learning relative to the children who received only the principle instruction. It was hypothesized that either (a) principle information was appropriate for some children, whereas procedure information was appropriate for other children; or (b) 


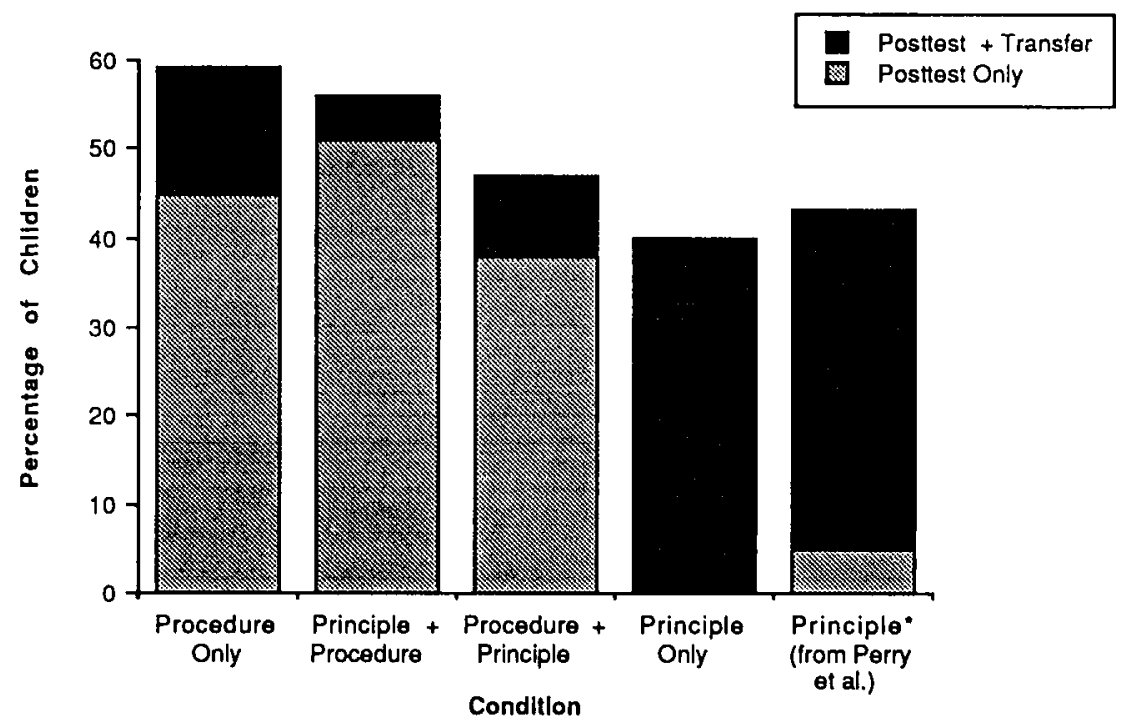

Figure 1. Percentage of children solving only the posttest problems or both the posttest and transfer problems correctly.

procedure information distracted children from understanding the principle of equivalence. The results from this study cannot lend support to the first hypothesis. The second hypothesis, however, could not be disconfirmed. In other words, the children receiving the procedure instruction seemed to be at a disadvantage in solving the transfer problems, even with the principle information available. Thus, future research should directly investigate and attempt to disconfirm the possibility that although some children who receive procedure-based instruction can learn those procedures, children do not analyze why the procedure worked, nor under what conditions it could be used.

\section{GENERAL DISCUSSION}

The aim of this research was to document how children's learning and transfer is affected by different types of instruction. It was demonstrated that the way information is presented can have a profound impact on wisat children learn. This research suggests that if children are provided with an easily accessible approach to solving a problem (in this case, any instruction containing a procedure), they may never consider the rationale underlying their problem-solving actions. Indeed, when children had an easily applied set of procedures at their disposal, they did not appear to consider why they were performing the procedures, even when explicitly presented with a principle.

Moreover, it seems important that children have a principle to guide them if 
they are to transfer. As Brown and Kane (1988) have pointed out, "In situations where learning can be organized around a guiding principle, transfer is determined by the extent that the subject is privy to that principle, through either discovery or instruction. . . Transfer is not automatic but depends upon insight into general principles" ( $p$. 495). In Study 1, it was demonstrated that the principle was critical to produce deeper learning, as evidenced by the ability to transfer.

It is interesting that procedural instruction maximally led to an ability to use the procedure, whereas principle-based instruction led to a richer conceptual understanding, which allows transfer to occur. A study by Sylva, Bruner, and Genova (1976), investigating the effects of different types of instruction on children's learning, may shed some light on why this occurred. Sylva et al. (1976) found that demonstrating directly to children how to solve a problem was just as effective as allowing children to have exposure to the problem-solving materials (i.e., they tested the effects of direct instruction vs. discovery learning). However, they also found that direct demonstration led to significantly fewer appropriate goal-directed responses. The children in the demonstration condition were likely to "opt out of the problem or go directly to the solution" (p. 252). On the other hand, the children who were not exposed to the procedure were likely to continue to attempt to find a solution even when they did not reach a solution. Sylva et al. concluded that the problem of the children who received demonstration should have been easy, but was not. In their view, observing the application (i.e., observing the correct procedure) may have created a narrow view of the problem. Consistent with Sylva et al., the research presented here illustrates that having access to a correct procedure may deter, rather than facilitate, understanding.

It is possible to take an educational message from the results of this study. If a teacher's goal is to have the students in his or her class solve a limited set of problems correctly, or to do well on certain types of tests, providing procedures may seem like the most efficient instructional strategy. However, no significant differences were found in the number of children in each condition who succeeded on the posttest (recall that the proportion of children passing this test ranged from $40 \%$ to $59 \%$; see Tables 2 and 3, pp. $456 \& 461$ ). Given that there was no advantage for presenting the procedure, even for the posttest problems, it can be argued that providing procedures cannot be the most efficient instructional strategy. On the contrary, relatively great benefits can be gained if children are presented with the principle and not with a procedure. Thus, if an educational message is to be taken from the results of this study, the message should be to provide children with underlying principles and let them figure out their own procedures for solving mathematical problems of the sort investigated here. It can be argued that educators should consider the power of principled instruction for an important mathematical concept such as equivalence: It would seem wiser 
to leave only some of the children in a classroom with a deep understanding rather than to leave them with a superficial understanding.

Two caveats are in order with respect to the quality of the principle provided in the instructions used here. First, it is possible to imagine other sorts of principlebased instructions. For example, the instructions could have been either more abstract (e.g., in terms of what the different sides of the equation mean) or more concrete (e.g., in terms of balance models). It is clear that there are many ways to communicate a principle-there is not necessarily one right way, although this possibility was not examined here. In any case, it is not the claim of this research that there is one true principle underlying each concept and presenting this would be the best way to promote understanding or growth of knowledge. Instead, the claim being made here is that the instructions used in these studies, based on the principle of equivalence, were significantly better at promoting growth of knowledge than instructions based on procedures for attaining mathematical equivalence.

Second, the instructions were very brief. Whereas it is certainly striking that children who were given both procedures and principles acquired procedures only, this was, after all, a very limited test of this possibility. It is possible to imagine more intensive and extensive instructional interventions, replete with examples, principles, procedures, and help at deriving a principle. In an educational setting where procedures and principles might be presented repeatedly over days or weeks, the outcome might conceivably be different. This possibility certainly should be pursued. It should be clear that this report does not provide a demonstration for the optimal type of instruction. Instead, it provides an indication that optimal learning of mathematical equivalence depends on being provided with a principle of equivalence, which is not tarnished with procedures.

Up to this point, the issue of individual differences has been ignored. Perhaps the most glaring omission has been the lack of discussion about whether some of the children may have been ready to learn, but others were not. Previous work (Perry et al., 1988) that used the same principle-based instruction used here found that some of the children appeared to be in a transitional knowledge state, and these children were more receptive to the principle-based instruction than children who were not in a transitional knowledge state. Perry et al. indexed this transitional knowledge state through observation of mismatches between children's speech, as they explained their problem solutions and the gestures that accompanied the spoken explanations. In the studies reported here, children's speech and gesture were also analyzed for mismatches. However, although similar proportions of children were identified as having transitional knowledge by the speech-and-gesture index (approximately $35 \%$ of the children), only the children in the principle-based instruction condition actually learned and evidenced transfer after instruction. Recall that very few, if any, of the children in the other conditions demonstrated these outcomes. Thus, it appears that when a 
child possesses transitional knowledge, he or she can only make transitions in understanding if appropriate instructional conditions are provided (for further discussion, see Perry, Church, \& Goldin-Meadow, in press). From this, it is likely that if all of the children had received only principle instruction, then those children in a transitional knowledge state would have demonstrated both learning and transfer. This lends further support to the hypothesis that most children will pay attention to, and perhaps be overwhelmed by a procedure, even if they could potentially make use of the principle-based instruction.

The results of the present research support previous work, including Brown and Kane (1988) and Hiebert and Lefevre (1986), which has found that principle instruction was the best method to foster children's understanding. The results of this research also support the work of those who claim that procedure instruction is fruitful (e.g., Corman, 1957), but qualifies the conclusions of the previous work. Specifically, procedure instruction can teach children to use correct procedures when they had been using incorrect procedures, but it is unlikely that procedure instruction leads to understanding beyond competence with those procedures.

It is clear that the way in which we teach children has a serious impact on what children learn. As Stodolsky (1988) pointed out: "The ways in which children learn subjects, in fact, may be a more important object of study than what they learn. Specific facts and content mastered in school are rather quickly forgotten. On the other hand, ideas about how you learn something . . . are more likely to endure" (p. 3). The lesson here is that if we provide children with tools for thinking, as opposed to procedures for doing, they will be more likely to think and reach a deeper understanding, and eventually devise and implement their own procedures.

\section{REFERENCES}

Behr. M., Erlwanger, S., \& Nichols, E. (1980). How children view the equal sign. Mathematics Teaching, 92, 13-15.

Brown. A.L., \& French. L.A. (1979). The zone of potential development: Implications for intelligence testing in the year 2000. Intelligence, 3, 255-273.

Brown, A.L., \& Kane, M.J. (1988). Preschool children can learn to transfer: Learning to learn and learning from example. Cognirive Psychology, 20, 493-523.

Brown, J.S.. \& Burton, R.R. (1978). Diagnostic models for procedural bugs in basic mathematical skills. Cognitive Science, 2, 155-192.

Chi, M.T.H., \& Bassok, M. (1989). Learning from examples via self-explanations. In L.B. Resnick (Ed.), Knowing. learning, and instruction (pp. 251-282). Hillsdale. NJ: Erlbaum.

Corman, B.R. (1957). The effect of varying amounts and kinds of information as guidance in problem solving. Psychological Monographs: General and Applied, $7 /$ (2. Whole No. 431).

Gick, M.L., \& Holyoak, K.J. (1987). The cognitive basis of knowledge transfer. In S.M. Cormier \& J.D. Hagman (Eds.), Transfer of learning: Contemporary research and applications ( $\mathrm{pp} .9-$ 46). New York: Academic.

Hiebert, J. (1986). Conceptual and procedural knowledge: The case of mathematics. Hillsdale. NJ: Erlbaum. 
Hiebert. J., \& Lefevre, P. (1986). Conceptual and procedural knowledge in mathematics: An introductory analysis. In J. Hiebert (Ed.), Conceptual and procedural knowledge: The case of mathematics (pp. 1-27). Hillsdale, NJ: Erlbaum.

Judd. C.H. (1908). The relation of special training to general intelligence. Educational Review, 36. $28-42$.

Kieran. C. (1980). The interpretation of the equal sign: Symbol for an equivalence relation vs. an operator symbol. Proceedings of the Fourth International Conference for the Psychology of Mathematics Education. 163-169.

Osgood, C.E. (1949). The similarity paradox in human learning: A resolution. Psychological Review, 56, 132-143.

Perry, M. (1985. April). Children's strategies in interpreting symbolic equivalence. Paper presented at the meeting of the Society for Research in Child Development, Toronto.

Perry. M. . Church, R.B., \& Goldin-Meadow. S. (1988). Transitional knowledge in the acquisition of concepts. Cognirive Development, 3, 359-400.

Perry, M., Church, R.B., \& Goldin-Meadow, S. (in press). Is gesture/speech misnatch a general index of transitional knowledge? Cognirive Development.

Stodolsky, S.S. (1988). The subject matters. Chicago: University of Chicago Press.

Sylva, K., Bruner, J.S., \& Genova, P. (1976). The role of play in the problem-solving of children 35 years old. In J.S. Bruner. A. Jolly. \& K. Sylva (Eds.), Play-lts role in development and evolution (pp. 244-257). New York: Basic Books.

Thomdike. E.L. \& Woodworth, R.S. (1901). The influence of improvement in one mental function upon the efficiency of other functions. Psychological Review, 8, 247-261, 384-395, 553564.

Thorndike, E.L. (1926). Measurement of intelligence. New York: Teachers College Press.

\section{APPENDIX}

Two types of problems ( 10 problems altogether) were used to test children's transfer of the principle of equivalence from the problems on which they were instructed.

\section{Multiplication Problems}

Two of the transfer problems instantiated a new operation: multiplication. Children were asked to solve these two problems:

$2 \times 4 \times 3=-\times 4$, and

$5 \times 2 \times 4=5 \times-$

\section{Alternative-Solution Problems}

Eight of the transfer problems were problems designed to determine which solutions the child would consider acceptable answers to particular problems. The instructions used for this portion of the task were as follows:

I'm going to show you some problems that a teacher gave her class. One of the problems was $4+6+3=-+3$. I'm going to show you some of the answers her students gave to this problem. What I want you to do is to pretend that you're the teacher and tell me which of the students you think solved the problem right and which solved the problem wrong. 
1. Bill put 13 in the blank. (Write 13 in the blank.) Is Bill right or wrong? Why? (If the child needs to be probed to get a response, say "If you were the teacher what would you tell Bill?")

2. Suzy put 16 in the blank. (Write 16 in the blank.) Is Suzy right or wrong? Why?

3. John put 4 in the blank. (Write 4 in the blank.) Is John right or wrong? Why?

4. Mary put 10 in the blank. (Write 10 in the blank.) Is Mary right or wrong? Why?

5. Sam put $4+6$ in the blank. (Write $4+6$ in the blank.) Is Sam right or wrong? Why?

There was another problem the teacher gave her students. It was $\mathbf{5}+\mathbf{2}+\mathbf{8}=\mathbf{5}$

+ -

1. Jerry put 15 as his answer. Was Jerry right or wrong? Why?

2. Jackie put 20 as her answer. Was Jackie right or wrong? Why?

3. Leslie put 10 as her answer. Was Leslie right or wrong? Why? 